\title{
Estudio preliminar del potencial solar en el campus ciudad universitaria de la UNAH
}

\author{
Marco Antonio Flores Barahona, Ramón Obed Escalón Gonzales ${ }^{1}$
}

\section{RESUMEN}

Con el objetivo de determinar el potencial del recurso solar en la Ciudad Universitaria, UNAH, en la azotea del edificio E1, se instaló un piranómetro CMP 6 para medir la radiación solar global en superficie horizontal, un Piranómetro SP Lite-2 para medir la radiación global en una superficie inclinada a $14^{\circ}$ con orientación al sur, un datalogger LOGBOX SD, todos ellos de la marca Kip \& Zonen y una estación meteorológica automática DAVIS. Los registros de los parámetros meteorológicos de interés se realizaron en intervalos de un minuto.

La data obtenida fue sometida a un análisis estadístico que incluyó, promedios diarios mensuales y anuales, desviación estándar y distribución de frecuencia entre otros.

Los datos mostrados en este estudio son los recopilados por los tres piranómetros. Considerando el factor tiempo de medición, la data de mayor confiabilidad es la de la estación meteorológica DAVIS, ya que ésta ha estado registrando datos durante tres años consecutivos, en cambio los datos obtenidos mediante los piranómetros Kip \& Zonen se han registrado únicamente durante ocho meses.

Este trabajo nos muestra que la radiación solar promedio diario anual disponible en la Ciudad Universitaria, $4.21 \mathrm{kWh} / \mathrm{m} 2$, es similar a los resultados obtenidos, para la Región Central del país mediante el Proyecto SWERA que nos indica un valor entre 4.5 y $5.0 \mathrm{kWh} / \mathrm{m} 2 / \mathrm{día}$, y con $4.869 \mathrm{kWh} / \mathrm{m} 2$ promedio diario, medidos con un piranómetro Eppley PSP en la UNAH en el período $1985-1990$ y $5.01 \mathrm{kWh} / \mathrm{m} 2$ en promedio diario para un período de 8 meses con un piranómetro Kip \& Zonen CPM 6.

Palabras clave: Potencial solar, piranómetro, datalogger, Generador FV de 230 kW, radiación promedio diario mensual, radiación promedio diario anual, radiación promedio horaria mensual, distribución de frecuencia.

\footnotetext{
${ }^{1}$ Instituto de Investigación en Energía/Sección de Energía, Escuela de Física, Facultad de Ciencias, Universidad Nacional Autónoma de Honduras, marco.flores@unah.edu.hn, marcoaflores@yahoo.com
} 


\section{ABSTRACT}

In order to determine the potential of the solar resource in the UNAH Campus on the roof of the E1 building, a CMP 6 pyranometer was installed to measure the global solar radiation on a horizontal surface, an SP Lite-2 Piranometer for measure the global radiation on a surface inclined at $14^{\circ}$ facing south, a LOGBOX SD data logger, all of them from the Kip \& Zonen brand and a DAVIS automatic weather station. The records of the meteorological parameters of interest were made in intervals of one minute. The data obtained was subjected to a statistical analysis that included daily and monthly averages, standard deviation and frequency distribution among others. The data shown in this study are those collected by the three pyranometers. Considering the measurement time factor, the data of greater reliability is that of the weather station DAVIS, since it has been recording data for three consecutive years, however the data obtained by the Kip \& Zonen pyranometers have been recorded only for eight months. This work shows us that the average daily solar radiation per year available in the UNAH Campus, $4.21 \mathrm{kWh} / \mathrm{m} 2$, is similar to the results obtained, for the central region of the country through the SWERA Project that indicates a value between 4.5 and $5.0 \mathrm{kWh} / \mathrm{m} 2 /$ day, and with $4.869 \mathrm{kWh} / \mathrm{m} 2$ daily average, measured with an Eppley PSP pyranometer in the UNAH in the period $1985-1990$ and $5.01 \mathrm{kWh} / \mathrm{m} 2$ in daily average for a period of 8 months with a Kip \& pyranometer Zonen CPM 6.

Key words: Solar potential, pyranometer, datalogger, 230 kW PV generator, monthly average daily radiation, annual average daily radiation, monthly average radiation, frequency distribution. 


\section{INTRODUCCIÓN}

El objetivo principal de este trabajo es la determinación del potencial solar en la ciudad universitaria, con el fin de estudiar la factibilidad económica de la instalación de un sistema solar fotovoltaico de $230 \mathrm{~kW}$ conectado a la red y capacitar a personal de la Sección de Energía de la Escuela de Física de la UNAH en el estudio del potencial in situ de la energía solar y en la preparación de estudios de factibilidad para generadores fotovoltaicos conectados a la red.

Este proyecto está enmarcado en el uso racional de la energía. El estudio se justifica desde dos puntos de vista fundamentales para la sostenibilidad económica y ambiental del consumo energético en el ámbito nacional:

1) Mediante la determinación del potencial solar in situ, se logra dimensionar y optimizar un generador solar fotovoltaico conectado a la red, cuyo fin es aprovechar el recurso solar disponible en la ciudad universitaria, reducir la dependencia, de la UNAH del sistema interconectado nacional, comprimir la factura del consumo eléctrico de la institución y en el marco del Decreto 138-2013 (Gaceta, 2013), el excedente de generación durante la Universidad permanece cerrada, se venderá de acuerdo a Ley. Con el fin de mejorar la rentabilidad del proyecto, es posible aprovechar el mecanismo de desarrollo limpio (MDL) u otro modelo enmarcado en la estrategia de mitigación del cambio climático.

2) Reducir las emisiones de gases de efecto invernadero causantes del cambio climático global, ser un modelo para la generación eléctrica distribuida con energía renovable.

\section{Principales fenómenos climatológicos que afectan a Honduras}

Como consecuencia de la ubicación geográfica de Honduras, nuestra atmósfera local es influenciada por diversos fenómenos climatológicos que afectan la radiación solar en nuestro territorio, entre ellos, los principales son: La zona de convergencia intertropical (ZCIT) que influencia el país desde comienzos de mayo a mediados de julio y de mediados de agosto a mediados de octubre, siendo las regiones sur, central, occidental-sur y una parte del occidente-norte, las más afectadas por este fenómeno; los frentes fríos se presentan a inicios de la segunda quincena de octubre hasta los últimos días de marzo o abril, afectan principalmente la región insular, norte, occidental-norte y el centro-norte; y, el anticiclón de las Bermudas que se presenta de la segunda quincena de julio a mediados de agosto, su efecto es notorio en la región 
sur, occidental-sur, central y occidental norte. La temporada de huracanes se presenta de junio a finales de octubre, época en la que se incrementa la precipitación en la zona norte, insular y occidental norte, con un poco de menor influencia en la zona central y sur.

\section{El contexto de la medición de la radiación solar del proyecto}

Las variaciones de la latitud en el territorio hondureño, la geografía, el tipo de vegetación, las condiciones atmosféricas y el clima local, son algunas de las causas por las que la energía solar incidente sobre Honduras, al igual que en todo el planeta, no sea uniforme en el contexto espacial y temporal.

"Honduras se encuentra en una de las regiones de mayor insolación del planeta, localizada en el amplio cinturón ecuatorial entre $15^{\circ}$ de latitud norte y $15^{\circ}$ de latitud sur, zona caracterizada por una alta humedad, gran nubosidad, leves variaciones estaciónales y elevada proporción de radiación difusa con un promedio anual total de 2,300 horas de brillo solar. La zona central del país tiene un promedio diario mensual de 6.7 horas" (Flores, Cartas Preliminares de Horas Sol de Honduras, 2000).

Proyectos de energía solar, como los sistemas fotovoltaicos domiciliares (SFVD) 0 para pequeños emprendimientos y aplicaciones, no requieren conocer con precisión el recurso solar disponible en el sitio donde se instalará un pequeño sistema de aprovechamiento del recurso solar.

Para los grandes sistemas de energía solar, es fundamental el máximo aprovechamiento de la energía incidente, mediante su óptimo dimensionamiento con el fin de obtener la máxima rentabilidad económica, por lo que es imprescindible conocer con precisión la disponibilidad real del recurso solar en el sitio específico. Información que únicamente es posible obtener mediante la medición directa del potencial solar in situ, con equipo de gran precisión, disponer de registros históricos de la insolación local y practicar un análisis estadístico a la data obtenida.

"La estación meteorológica experimental del Departamento de Física del centro universitario de estudios generales de la Universidad Nacional Autónoma de Honduras en Tegucigalpa, registró datos de radiación solar, desde 1985 hasta 1990, con un promedio diario mensual de $4.869 \mathrm{kWh} / \mathrm{m} 2$. Haciendo algunos cálculos y consideraciones muy sencillas, y tomando ese único dato como referencia nacional, teóricamente encontramos que nuestro territorio recibiría anualmente $547 \mathrm{TWh}$. Tomando en consideración que la producción nacional de energía eléctrica en 1996 fue de 3.05 
TWh, observamos que el consumo nacional de energía eléctrica representa únicamente el $0.56 \%$ de la energía solar que recibe el país." (Flores, Atlas Heliofánico de Honduras, 2000). En 2012 la generación eléctrica total fue de 6,370.9 GWh (6.4 TWh) lo que representa apenas el $1.16 \%$ de la energía solar incidente en el país.

El proyecto solar and Wind Energy Resources Assesstment (SWERA), mediante información satelital, determinó que la zona central del país, tiene un potencia solar entre 4.5 y $5.0 \mathrm{kWh} / \mathrm{m} 2 /$ día (Flores, Reporte de Energía Proyecto SWERA, 2008), rango que coincide con el promedio diario mensual (4.869 kWh/m2/día) medido en la UNAH, mediante un piranómetro Eppley PSP (Flores, Atlas Heliofánico de Honduras, 2000), dato que valida los resultados del Proyecto SWERA.

Los datos de energía solar para una superficie horizontal, en el marco del Proyecto No. 092-2012 Determinación del Potencial Solar en la Ciudad Universitaria y estudio de factibilidad de una planta solar fotovoltaica, fueron registrados mediante una estación meteorológica automática DAVIS durante tres años. Período para el que obtuvimos una insolación promedio diario mensual de $4.21 \mathrm{kWh} / \mathrm{m} 2 /$ día. Con el piranómetro Kip \& Zonen CMP 6 obtuvimos $5.01 \mathrm{kWh} / \mathrm{m} 2$ en promedio diario mensual para un período de 8 meses y con el piranómetro Kip \& Zonen SP Lite-2 inclinado $14^{\circ}$ y orientación sur obtuvimos un promedio diario mensual de $4.98 \mathrm{kWh} / \mathrm{m} 2$ también para un período de 8 meses. La diferencia obtenida para cada uno de los equipos, en principio puede ser gracias a que los periodos durante los cuales se midió el recurso no son iguales, también existe una diferencia en cuanto a la precisión del equipo para medir la radiación solar y el efecto de la temperatura en cada uno de ellos. En ese sentido, el piranómetro de mayor calidad es el Kip \& Zonen CMP 6.

\section{Procedimiento}

Para la medición del recurso solar en el campus de la ciudad universitaria, UNAH, se instaló un piranómetro Kip \& Zonen CMP 6, para medir la radiación solar global en superficie horizontal, un Piranómetro Kip \& Zonen SP Lite-2 para medir la radiación solar en superficie inclinada a la latitud de la Ciudad Universitaria $\left(14^{\circ}\right)$, un datalogger Kip \& Zonen LOGBOX SD con una memoria SDM de 512 MB, ocho (8) entradas analógicas y cuatro (4) digitales, que registró datos cada minuto y una estación meteorológica automática DAVIS con sensores, con una precisión de $+/-5 \%$, para medir radiación solar, radiación UV, presión atmosférica, humedad, temperatura ambiente, precipitación, velocidad y dirección del viento, entre otros parámetros meteorológicos. El equipo de medición, gracias a la facilidad y disponibilidad de un sitio donde instalarlo, se ubicó en la azotea (cuarto piso, unos $12 \mathrm{~m}$ sobre el nivel del 
suelo) del edificio E1, en las instalaciones del Instituto de Investigación en EnergíaSección de Energía, Facultad de Ciencias. Todo el equipo fue instalado de acuerdo con los respectivos manuales de instalación de cada uno de ellos (normas internacionales).

La información obtenida nos proporciona información sobre la radiación total mensual y anual en unidades Hsd ( $1 \mathrm{Hsd}=1 \mathrm{kWh} / \mathrm{m} 2$ ), indicando sus valores máximos y mínimos. También, la radiación promedio diario mensual, el promedio diario anual y el promedio diario mensual durante los años de registro, además de los valores diarios máximos y mínimos, promedios horarios mensuales y la distribución de frecuencia acumulada. Valores que nos fueron de utilidad para determinar el potencial solar en la ciudad universitaria.

\section{Resultados e Interpretación de la Data de Insolación}

La data de insolación registrada por la estación meteorológica automática DAVIS para el período mayo de 2010 a abril de 2013 se muestran en la Tabla 1, donde figura el promedio diario mensual en Hsd , los promedios mensuales, anuales y el promedio durante el período. También se muestran los valores máximos y mínimos mensuales y anuales; y el valor instantáneo máximo.

En la Tabla 1 y Gráfico 1 observamos que, para Tegucigalpa, zona central del país, los meses de mayor insolación coinciden con la época seca, de marzo a abril, de mayo a julio se reduce la insolación gracias a la presencia de la ZTIC en el istmo centroamericano, que produce la estación lluviosa, posteriormente en julio-agosto se presenta un período seco, conocido como veranillo. Entre agosto y septiembre nuevamente sube la ZTIC provocando una nueva estación lluviosa. En octubre se inician los frentes fríos que afectan la zona central de octubre a enero con bastante intensidad y con menor intensidad hasta marzo 0 abril. La temporada de huracanes se inicia en junio y termina a finales de octubre (Flores, Cartas Preliminares de Horas Sol de Honduras, 2000).

En la Tabla 2 y Gráfico 2 se presentan los totales mensuales y anuales de insolación, donde los totales de radiación solar mensual, de acuerdo a la explicación anterior, tienen una variación tal como se esperaba.

\footnotetext{
${ }^{1} \mathrm{Hsd}=1 \mathrm{kWh} / \mathrm{m} 2$
} 

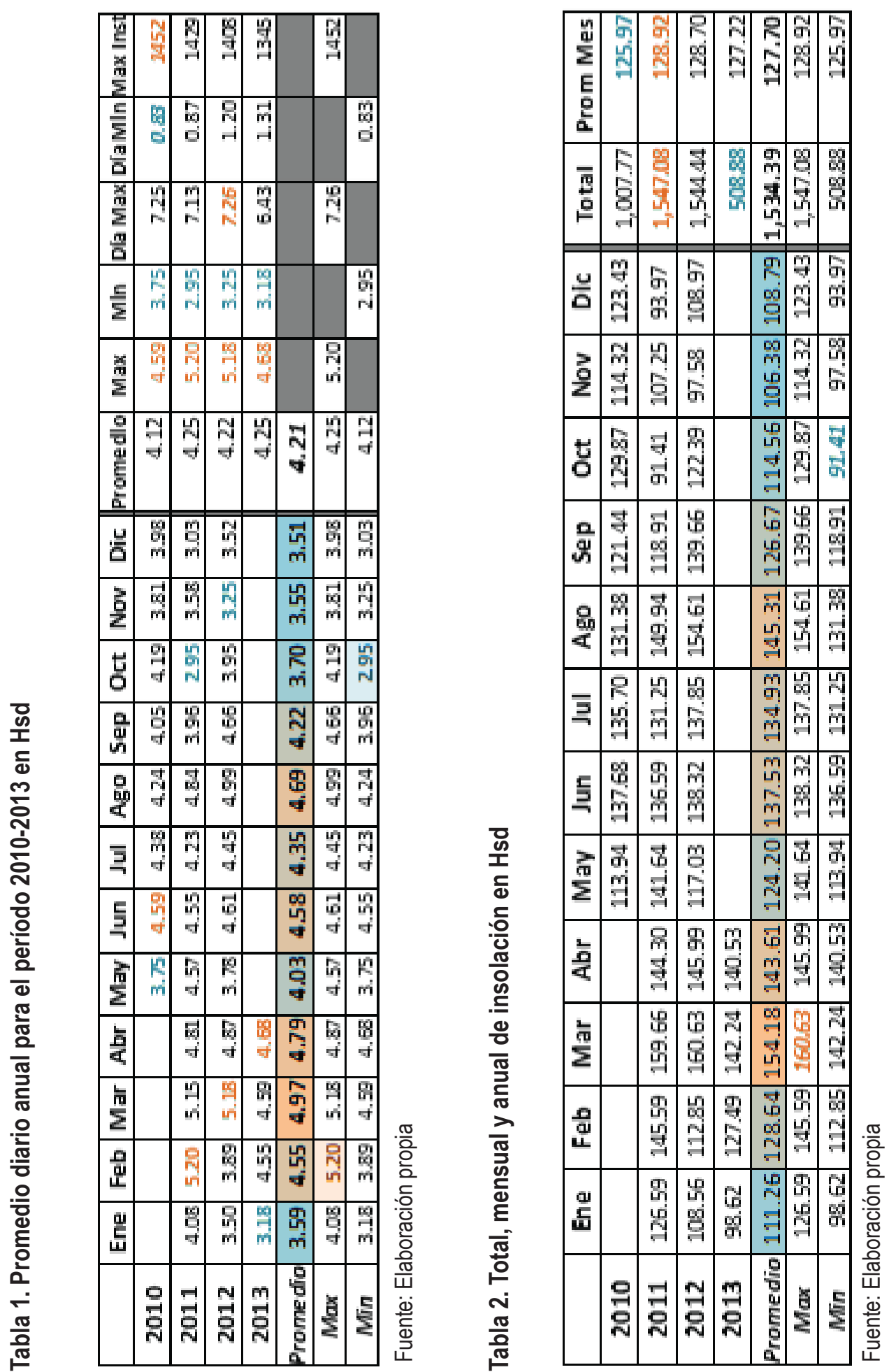


\section{Gráfico 1. Promedio diario mensual}

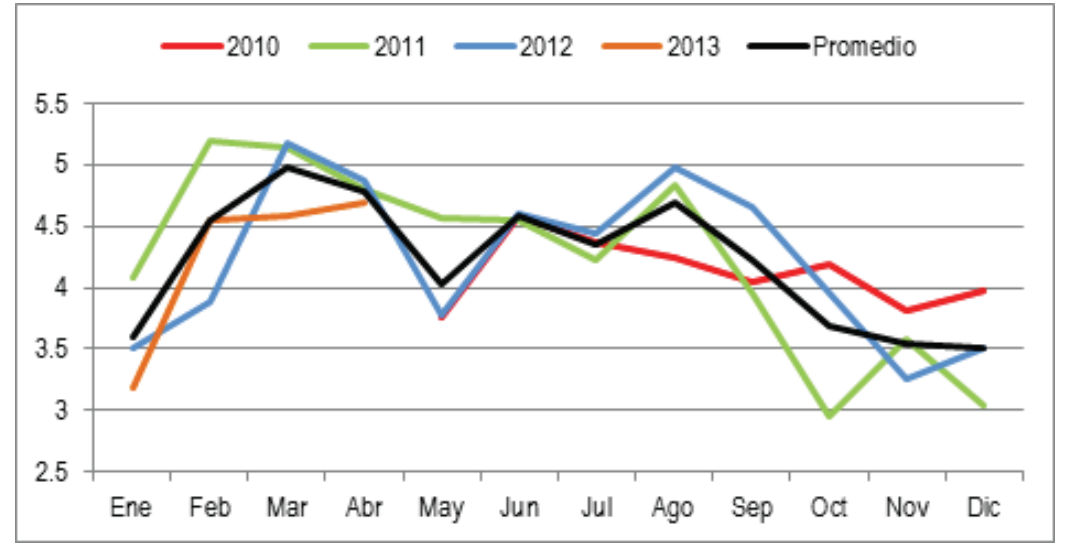

Fuente: Elaboración propia

Gráfico 2. Total, mensual y anual de insolación

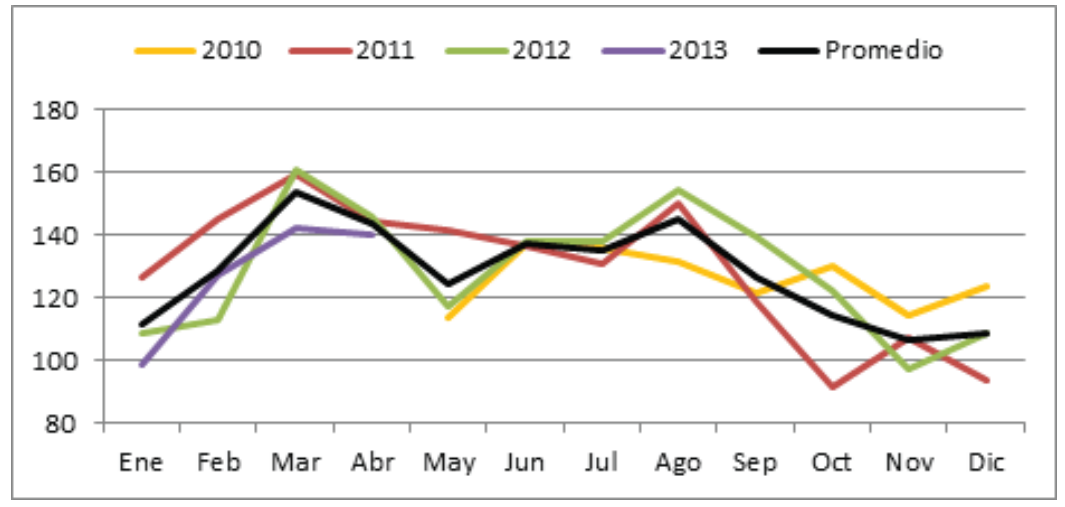

Fuente: Elaboración propia

En la Tabla 3 y Gráfico 3 se observa la distribución horaria mensual promedio anual de la radiación. Este factor es muy importante para determinar las horas y cantidad de energía eléctrica que un generador eléctrico puede entregar al sistema, lo que determina el factor de planta horaria de una instalación solar fotovoltaica conectada a la red. 


\begin{tabular}{|c|c|c|c|c|c|c|c|c|c|}
\hline 뎡 & 응 & 음 & 응 & 8 & 영 & 응 & 응 & 8 & 옹 \\
\hline 点 & 襖 & 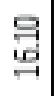 & 옴 & 영 & 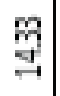 & 흄 & 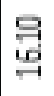 & 옹 & 哭 \\
\hline 臬 & $\begin{array}{l}\text { 号 } \\
\text { 윽 }\end{array}$ & 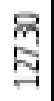 & $\begin{array}{l}\text { 晕 } \\
\text { 总 }\end{array}$ & $\begin{array}{l}\mathrm{G} \\
\mathrm{g} \\
\rightarrow\end{array}$ & 莺 & $\begin{array}{l}\text { 号 } \\
\text { m }\end{array}$ & 㝵 & $\begin{array}{l}\text { 吕 } \\
\text { g }\end{array}$ & $\frac{2}{5}$ \\
\hline 岳 & $\begin{array}{l}\text { 号 } \\
\text { 胥 }\end{array}$ & 膏 & $\begin{array}{l}\text { 志 } \\
\text { : } \\
\text { g }\end{array}$ & $\begin{array}{l}\text { m } \\
\text { 号 } \\
\text { 足 }\end{array}$ & $\begin{array}{l}\mathbb{8} \\
\mathbb{8}\end{array}$ & ำ & 営 & $\begin{array}{l}\text { 号 } \\
\text { 焉 }\end{array}$ & $\begin{array}{l}\text { 总 } \\
\text { 范 }\end{array}$ \\
\hline 독 & $\begin{array}{l}9 \\
8 \\
8\end{array}$ & 光 & 皆 & ㄹ. & 学 & 혹 & 총 & $\begin{array}{l}9 \\
8 \\
8\end{array}$ & $\begin{array}{l}\text { 록 } \\
\text { 응 }\end{array}$ \\
\hline 들 & ज्ञ & 园 & $\begin{array}{l}\mathscr{g} \\
\mathbb{M}\end{array}$ & 题 & 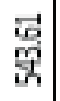 & 总 & 总 & 总 & 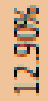 \\
\hline 국 & $\begin{array}{l}\text { ○ } \\
\text { 罳 }\end{array}$ & 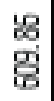 & \begin{tabular}{l}
$\mathbb{8}$ \\
\multirow{7}{6}{}
\end{tabular} & $\begin{array}{l}m \\
m \\
m\end{array}$ & $\begin{array}{l}\text { व̃ } \\
\text { 봄 }\end{array}$ & 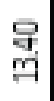 & $\begin{array}{l}\mathrm{m} \\
\mathrm{m}\end{array}$ & $\begin{array}{l}6 \\
\text { 总 }\end{array}$ & $\begin{array}{l}\text { 总 } \\
\text { 岌 }\end{array}$ \\
\hline 들 & 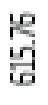 & 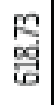 & $\begin{array}{l}\mathbb{2} \\
\mathbb{8} \\
6\end{array}$ & क्ष & $\begin{array}{l}8 \\
\mathrm{~g} \\
6\end{array}$ & 嗯 & 总 & 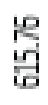 & $\begin{array}{l}\text { 瓷 } \\
\text { 足 }\end{array}$ \\
\hline 동 & 菑 & $\begin{array}{l}\text { 突 } \\
\text { 茎 }\end{array}$ & 总 & $\begin{array}{l}\mathscr{8} \\
\mathscr{8} \\
\end{array}$ & $\begin{array}{l}\text { ज } \\
\text { 名 }\end{array}$ & $\begin{array}{l}8 \\
6 \\
6\end{array}$ & $\begin{array}{l}\text { 总 } \\
\text { 㿣 }\end{array}$ & 焉 & $\begin{array}{l}\text { 总 } \\
\text { mె }\end{array}$ \\
\hline 등 & $\begin{array}{l}\vec{G} \\
\text { 要 }\end{array}$ & $\begin{array}{l}m \\
\stackrel{9}{\xi} \\
\text { जี }\end{array}$ & 萝 & $\begin{array}{l}\infty \\
m \\
8 \\
8\end{array}$ & 总 & $\begin{array}{l}\nexists \\
⿱ 士 \\
\ddots\end{array}$ & 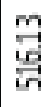 & $\begin{array}{l}\infty \\
\text { 盟 }\end{array}$ & 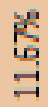 \\
\hline $\bar{\infty}$ & $\begin{array}{l}\text { 是 } \\
\text { 学 }\end{array}$ & $\begin{array}{l}\stackrel{m}{m} \\
\stackrel{m}{m}\end{array}$ & $\begin{array}{l}\text { 昰 } \\
\text { ले }\end{array}$ & $\begin{array}{l}m \\
\overrightarrow{2}\end{array}$ & m & \begin{tabular}{l}
$\infty$ \\
$\approx$ \\
\hdashline
\end{tabular} & $\begin{array}{l}m \\
m \\
m \\
m\end{array}$ & $\begin{array}{l}\text { m } \\
\text { 总 }\end{array}$ & 発 \\
\hline$\frac{c}{N}$ & 学 & $\stackrel{Ð}{\Xi}$ & $\begin{array}{l}m \\
\text { g్ } \\
g\end{array}$ & 号 & $\begin{array}{l}\text { 孝 } \\
\text { 岁 }\end{array}$ & 옴 & 总 & $\begin{array}{l}\text { 음 } \\
\text { 임 }\end{array}$ & 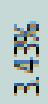 \\
\hline 悤 & 里 & $\underset{8}{\stackrel{9}{9}}$ & 粗 & ติ & 壳 & 要 & 党 & ma & $\begin{array}{l}\text { 梁 } \\
\text { 号 }\end{array}$ \\
\hline 든 & ఫ & ్ㅁㅇ & 븡 & 8 & $\exists$ & $\dddot{2}$ & తి & 8 & 总 \\
\hline & 임 & 롬 & ్ㅗㄱ & 롬 & $\begin{array}{l}\text { 옿 } \\
\text { 튼 } \\
\text { 은 }\end{array}$ & 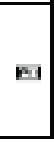 & $\stackrel{\text { 奠 }}{\Sigma}$ & 들 & de \\
\hline
\end{tabular}

苝 8

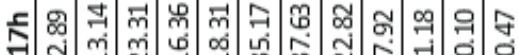

ธ

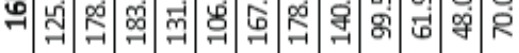

ร ฤ ๆ

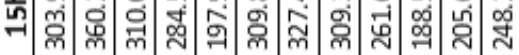

× ตี่

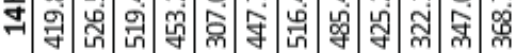

ร

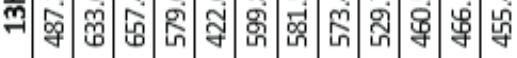

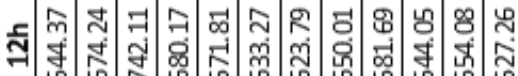

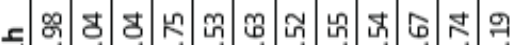

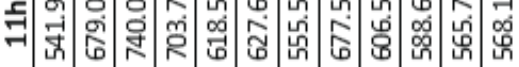

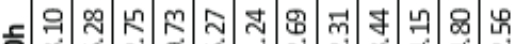

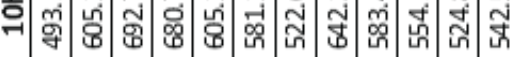

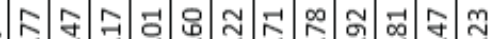

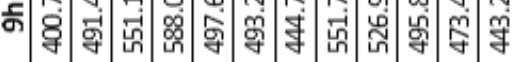

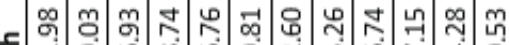
ळ

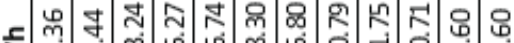

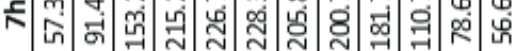

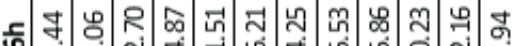
ल ปู่

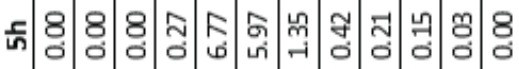
원 


\section{Gráfico 3. Radiación horaria}

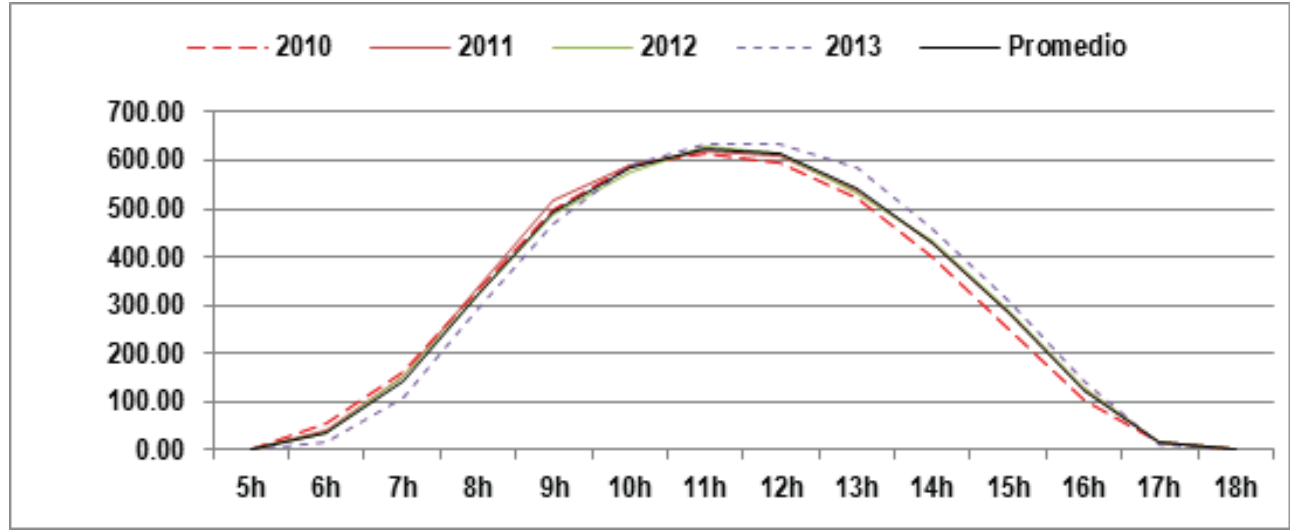

Fuente: Elaboración propia

Observamos que la mayor capacidad de un generador FV en la Ciudad Universitaria estaría en el horario de 9:00 AM a 2:00 PM, que concuerda con las horas de mayor consumo energético, coincidiendo con el primer pico de consumo energético en Honduras, período en el que el precio de la energía eléctrica es mayor porque en ese pico entran las plantas de generación eléctrica a Diesel.

El sensor de radiación solar de la estación meteorológica automática DAVIS, tiene una precisión de $\pm 5 \%$. Este rango de tolerancia de las mediciones, dependiendo del número de datos, tiene importancia en los valores finales. Cuando el número de lecturas es pequeño (en especial para un promedio con pocos datos) puede ser importante, pero a medida que el número de muestras se incrementa, esta incertidumbre tendrá menor peso en un valor consolidado. Para determinar el potencial solar en Ciudad Universitaria, se analizaron 3 años de datos (365 días al año, 1,440 muestras diarias) obteniendo 1,576,800 lecturas de la radiación global para este trabajo; para esta cantidad de muestras la incidencia del equipo de $\pm 5 \%$ no tendrá un efecto considerable que afecte el valor promedio.

Se hicieron muestreos aleatorios de variaciones en los datos, en un total de 1,800 muestras, el promedio de variación en los datos nunca superó el $0.1 \%$ de su valor central. Considerando que las lecturas usadas en este estudio son casi mil veces mayores al muestreo piloto, podemos inferir que la tolerancia del aparato no introducirá un error considerable en los resultados del experimento.

En el Gráfico 4 se muestra el consumo promedio entre semana del Edificio E1, donde observamos las horas de mayor consumo eléctrico. 


\section{Gráfico 4. Potencia activa trifásica promedio de lunes a viernes en kWh}

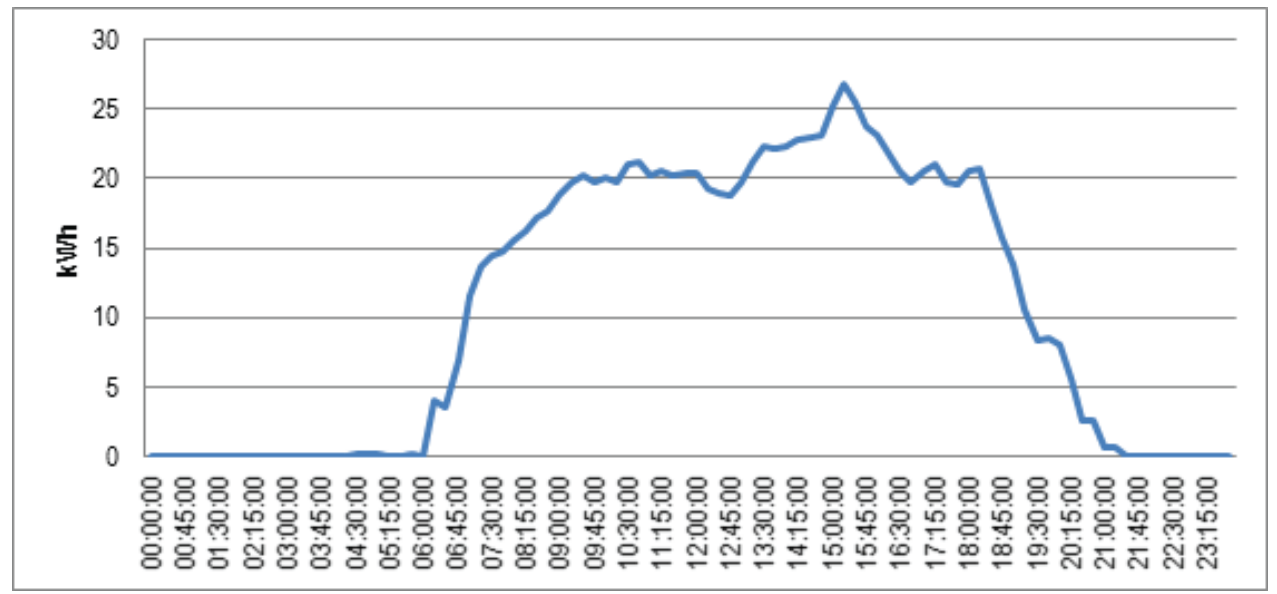

Fuente: Elaboración propia

La Tabla 4 y Gráfico 5 nos presentan la insolación promedio horario mensual total durante el período de medición del recurso solar.

\section{Gráfico 5. Promedio horario mensual (Wh/m2)}

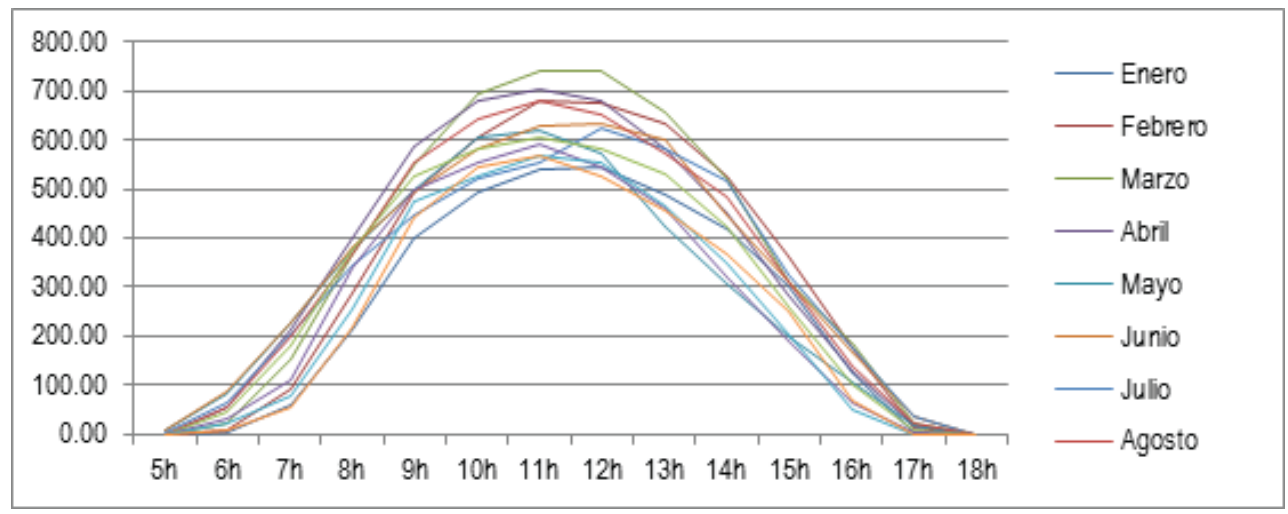

Fuente: Elaboración propia

La Tabla 5 nos indica la distribución de frecuencia de la insolación, es decir el número de eventos en los cuales tuvimos un cierto nivel (rango) de radiación solar. 
Tabla 5. Distribución de frecuencia y frecuencia acumulada

\begin{tabular}{|c|c|c|c|c|}
\hline Rango (Hsd) & Frecuencla & \% Frecuencla & Frec. Acumulada & \%Frec. Acumulada \\
\hline$<1$ & & & & \\
\hline $1.0-1.5$ & & & 8 & $0.74 \%$ \\
\hline $1.5-2.0$ & 35 & $3.22 \%$ & 43 & $3.96 \%$ \\
\hline $2.0-2.5$ & 70 & $6.45 \%$ & 113 & $10.41 \%$ \\
\hline $2.5-3.0$ & 75 & $6.91 \%$ & 188 & $17.31 \%$ \\
\hline $3.0-3.5$ & 102 & $9.39 \%$ & 290 & $26.70 \%$ \\
\hline $3.5-4.0$ & 146 & $13.44 \%$ & 436 & $40.15 \%$ \\
\hline $4.0-4.5$ & 140 & $12.89 \%$ & 576 & $53.04 \%$ \\
\hline $4.5-5.0$ & 141 & $12.986 \%$ & 717 & $66.02 \%$ \\
\hline $5.0-5.5$ & 129 & $11.868 \%$ & 846 & $77.90 \%$ \\
\hline $5.5-6.0$ & 103 & $9.48 \%$ & 949 & $87.38 \%$ \\
\hline $6.0-6.5$ & 77 & $7.09 \%$ & 1026 & $94.48 \%$ \\
\hline $6.5-7.0$ & 39 & $3.59 \%$ & 1065 & $98.07 \%$ \\
\hline $7<$ & 21 & $1.93 \%$ & 1086 & $100.00 \%$ \\
\hline
\end{tabular}

Fuente: Elaboración propia

En el Gráfico 6 observamos la distribución de frecuencia de la insolación.

\section{Gráfico 6. Distribución de frecuencia}

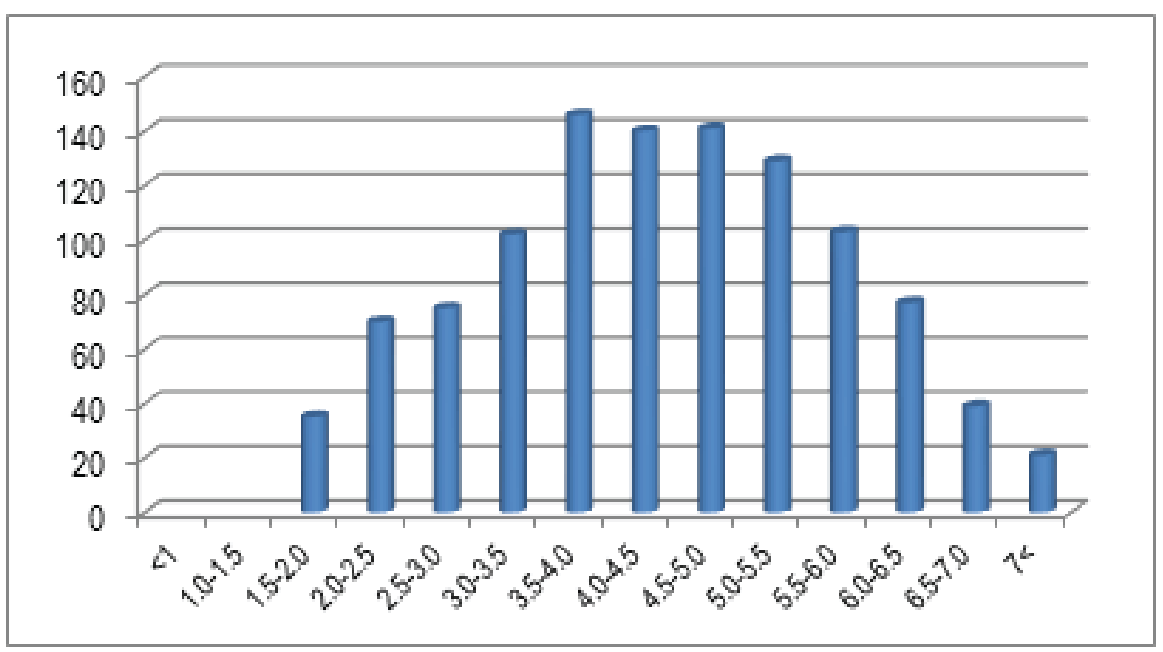

Fuente: Elaboración propia 


\section{CONCLUSIONES}

Los resultados del estudio muestran que el potencial solar disponible en el Campus de la Ciudad Universitaria es $4.21 \mathrm{kWh} / \mathrm{m} 2 /$ día, $+/-5 \%$ de error del equipo, valor que es adecuado para la generación eléctrica. No necesariamente el más apropiado para una central fotovoltaica de varias decenas o centenas de Mega Watts (MW) pero si para un proyecto de varias decenas o pocas centenas de kilo Watts (kW); por ejemplo, es apropiado para el tamaño de una central FV que establece el Decreto 138-2013, Ley de Incentivos a la Generación Eléctrica con Recursos Renovables. Donde se implantan reglas de mercado para centrales FV de hasta $250 \mathrm{kWp}$ privadas o en instituciones estatales centralizadas o descentralizadas, como es el caso de la UNAH. Nuestros resultados, de acuerdo con el relativamente poco tiempo de registro de la insolación son adecuados para alimentar una central fotovoltaica de mayor dimensión que la propuesta. El Edificio E1 en promedio semanal tiene un consumo máximo en potencia de $26.8 \mathrm{~kW}$ a la hora pico, momento en que, la energía solar aun es importante. En su azotea se pueden instalar $82 \mathrm{kWp}$.

\section{AGRADECIMIENTOS}

Los autores desean agradecer a la Dirección de Investigación Científica y Posgrados de la Universidad Nacional Autónoma de Honduras por su confianza en el Proyecto y por su apoyo financiero para el desarrollo de éste; así mismo, a Gerardo López por su oportuna colaboración y apoyo en varias fases del Proyecto.

\section{REFERENCIAS BIBLIOGRAFÍCAS}

Akinoglu B. G. (1991), A Review of Sunshine-Based Models Used to Estimate Monthly Average Global Solar Radiation, Renewable Energy, Vol. 1, p. 479-497.

Duffie J. A. and Beckman W. A. (1980), Solar Engineering of Thermal Processes, Wiley Interscience Publication, EEUU.

Flores, M. (2000). Atlas Heliofánico de Honduras. Tegucigalpa, Honduras.

Flores, M. (2000). Cartas Preliminares de Horas Sol de Honduras. (C. Estrada, Ed.) Proceeding of the Millenium Solar Forum 2000, 633-640.

Flores, M. (2008). Reporte de Energía Proyecto SWERA, SWERA, Tegucigalpa. 
Garg H. P. and Garg S. N. (1993), Measurement of Solar Radiation - I, Radiation Instrument, Renewable Energy, Vol. 3, p. 321-333.

Grossi Gallegos H. (1996), Curso de Solarimetría, Sección de Energía, Departamento de Física, Centro Universitario de Estudios Generales. Universidad Nacional Autónoma de Honduras.

Iqbal M. (1983), An Introduction to Solar Radiation, Academic Press, Canada.

Kreyszig E. (1970), Introductory Mathematical Statistics. Principles and Methods, John Wiley and sons, Inc. EEUU.

La Gaceta. (01 de agosto de 2013). Reforma a la Ley de Promoción a la Generación Eléctrica con Recursos Renovbales. (No. 31,191), págs. 2-13.

Levin J. (1979), Fundamentos de Estadística, Segunda Edición, Harla, México.

Ramage J. (1987), Energy a Guide Book, Oxford University Press, England.

Ruiz Hernández V. (1996), Apuntes Maestría en Técnicas de Energía Renovable en la Ingeniería, Arquitectura y Agricultura, Universidad Internacional de Andalucía, La Rábida, España.

Vernich L. and Zuanni F. (1996), About the Minimum Number of Years Required to Stabilize the Solar Irradiation Statistical Stimates, Solar Energy, Vol. 57, p. 445-448.

Zúniga E., (1989), La Estructura y el Comportamiento de la Atmósfera, Honduras. 